\title{
Systematic Errors in Long Baseline Oscillation Experiments
}

\author{
D.A.Harris ${ }^{\mathrm{a}}$ \\ ${ }^{a}$ Fermi National Accelerator Laboratory, \\ P.O. Box 500, Batavia, Illinois, 60510 USA
}

This article gives a brief overview of long baseline neutrino experiments and their goals, and then describes the different kinds of systematic errors that are encountered in these experiments. Particular attention is paid to the uncertainties that come about because of imperfect knowledge of neutrino cross sections and more generally how neutrinos interact in nuclei. Near detectors are planned for most of these experiments, and the extent to which certain uncertainties can be reduced by the presence of near detectors is also discussed.

\section{Current Long Baseline Experiments}

The current set of long baseline neutrino experiments hope to verify the oscillation framework, make ever more precise measurements of the neutrino mass squared differences through muon neutrino disappearance measurements, and search for the last unmeasured leptonic mixing angle through muon to electron neutrino transitions.

If in fact transitions from muon neutrinos to electron neutrinos are found in any of the current or next generations of long baseline oscillation experiments, then the field will focus on determining the neutrino mass hierarchy to see if neutrino masses are organized the way the charged fermions are, and also to search for CP violation. In the quark sector, CP violation is very small, but in the lepton sector, CP violation may be large, and may even be the source of the baryon asymmetry of the universe.

\subsection{Neutrino Beamlines}

The goal of seeing neutrino oscillations on a terrestrial distance scale has driven neutrino beamline design towards lower neutrino energies, compared to the higher energy goals of the previous generation of neutrino experiments. The K2K neutrino beamline operated with a mean neutrino energy of about $1.4 \mathrm{GeV}[1]$. The T2K neutrino beamline, currently under construction, has been designed to run at a mean off-axis neutrino energy of about $700 \mathrm{MeV}$, pointed some 2.5 degrees away from the Super-Kamiokande detector [2]. The NuMI beamline, which is currently producing 3$6 \mathrm{GeV}$ neutrinos for the MINOS experiment on the beamline axis, will also provide $2 \mathrm{GeV}$ neutrinos at about $15 \mathrm{mrad}$ off axis for the NOvA experiment later this decade [3]. The highest energy neutrino beamline that is doing long-baseline oscillation measurements is CNGS-because the experiments OPERA and ICARUS hope to measure $\nu_{\tau}$ appearance through the charged current channel, the neutrino energies must be well above the $3.5 \mathrm{GeV}$ threshold. The CNGS beamline produces a mean neutrino energy of about $25 \mathrm{GeV}$ [4].

\subsection{Neutrino Detectors}

The current generation of neutrino detector design spans a broad range of technologies, corresponding to the broad range of oscillation physics goals and energies listed above. The 5.4 kton MINOS detector, which is optimized for $\nu_{\mu}$ charged current measurements, consists of magnetized steel/ solid scintillator sandwhiches, where the longitudinal segmentation of the steel is $2.54 \mathrm{~cm}$, and the longitudinal segmentation of the scintillator is $1 \mathrm{~cm}$ [5]. This makes it ideal for a compact detector that measures both the presence and energy of charged current neutrino interactions, but makes separation between electron neutrino charged current events and neutral current interactions quite challenging. The 
XX kton NOvA detector, optimized for electron neutrino detection, is a segmented liquid scintillator detector whose longitudinal (transverse) segmentation is $6 \mathrm{~cm}(3.8 \mathrm{~cm})[6]$. The 50 kton Super-Kamiokande detector serves as the far detector for both the $\mathrm{K} 2 \mathrm{~K}$ and $\mathrm{T} 2 \mathrm{~K}$ experiments, and is optimized for low energy interactions: it is a water Cerenkov detector, whose size and photo-cathode coverage give it excellent energy resolution for both muon and electron neutrino quasielastic events [7].

The CNGS beamline, which produces the highest energy neutrinos, can afford to have the most fine-grained neutrino detectors because of the higher neutrino cross section at higher energies. The OPERA experiment will use 1.8 kton of leademulsion sandwiches followed by tracking and hadronic calorimetry to contain events [8]. The thickness of the lead in the sandwiches is only $1 \mathrm{~mm}$, which allows one to track the development of the electromagnetic shower as well as see the kink in the $\tau$ decay, for a large fraction of $\nu_{\tau}$ charged current events. The ICARUS experiment will use a 3 kton Liquid Argon TPC with $3 \mathrm{~mm}$ wire spacing, which like OPERA allows excellent tracking of electromagnetic showers from $\nu_{e}$ charged current events, and from $\tau \rightarrow e$ decays [9].

\subsection{Systematic Uncertainties}

Although the beam-lines and detectors described above span a broad range of energies and technologies, the systematic uncertainties these experiments will have to face can be classified in the same way. For all of these experiments, there will be uncertainties on the incident neutrino flux, the neutrino interaction model, and the effects of the detector on the neutrino event selection and energy reconstruction. Although most of the long-baseline neutrino experiments described here will have near detectors, the near detector can at best measure a combination of all of these effects, and different systematic effects will translate differently from the near to the far detector.

The neutrino flux uncertainties have two different sources: one is the geometry and focusing strength of the magnets in the beamline, but the more important uncertainty (at present) is in the spectrum of hadrons produced when the primary protons strike the target to make the secondary pions and kaons. In particular, the pion to kaon production ratio gives an important constraint on the high energy electron neutrino backgrounds in an experiment. Also, the transverse momentum distribution of the created secondaries will affect the neutrino energy distributions at the near and far detectors, since the focusing system cannot focus all transverse momenta equally.

Other systematic uncertainties come from the fact that the detector response is not known perfectly. In particular, if event selection and background rejection for a given analysis is not known adequately, then this corresponds to an uncertainty in the number of background and signal events that a far detector would see. Also, the energy response of a detector becomes important for measurements of the mass squared splitting, which require precise measurement of the muon neutrino disappearance probability versus neutrino energy. Translating between the true incoming neutrino energy and the observed energy in a neutrino detector, even for charged current events where all the final state particles are charged, is far from trivial.

Finally, uncertainties in the way neutrinos interact in a detector provide systematic biases as well, especially when convoluted with flux and detector response uncertainties. In general, detector efficiency and energy response are highly dependent on the type of interaction (Quasi-elastic, resonance, and Deep Inelastic scattering), and so uncertainties in the relative ratios of those processes again lead to uncertainties in the overall energy scale of a detector measurement, selection efficiency, and background rejection.

By using near detectors, many experiments try to remove these biases (by effectively applying them twice) but this only works effectively if the relative populations of neutrino events are the same between near and far detectors. For most near detector locations considered the neutrino fluxes $\left(\nu_{\mu}\right.$ and $\nu_{e}$ both) look different near to far, resulting in a different composition of processes (quasi-elastic, resonance, deep inelastic scattering, for example). A much more significant difference comes from the fact that muon neutrinos 
have a large disappearance probability for most long-baseline experiments. This means that the flavor distributions of the charged current events will be dramatically different between any near and far detector.

\subsection{Near Detectors}

There are many different strategies being pursued for far detector designs, but there are in fact even more strategies being considered for near detectors. In general they can be broken into two categories.

One option for a near detector design is to make the near detector as similar as possible to the far detector, if not identical. The argument for this is that if the detector technologies are identical, then uncertainties in the detector efficiency and reconstruction will cancel in the comparison of near to far events. However, as stated above, because the neutrino compositions are so different near to far, there is only partial cancellation. Furthermore, because the near and far detectors are necessarily very different sizes, fiducial mass and leakage in the near detector must be carefully modeled. Finally, the rates in the near detector are so much higher than in the far detector that often different electronics must be employed, and again care must be taken to understand the differences that are introduced.

A different option for a near detector is one that is much more segmented and fine-grained. This strategy means that one must try to measure the fluxes and cross sections as independently as possible, and then use that information to constrain the detector simulation so that the information is correctly extrapolated to the far detector. The concern with this strategy is that the detector simulation must accurately predict the detector response. Because the near and far detectors are not necessarily of the same target material, a part of the near detector must include some of the same target material so that nuclear effects on the cross sections (signal and background both) can be taken into account.

Table 1 gives a list of current neutrino experiments as well as their far and near detector technology choices.
Table 1

Survey of experiments, their far detectors, and their near detector strategies

\begin{tabular}{ll}
\hline \hline Experiment & Near Detector Strategy \\
\hline K2K [10] & Several, one identical \\
\hline MINOS [5] & identical, but faster electronics \\
\hline OPERA & No Near Detector \\
\hline ICARUS & No Near Detector \\
\hline T2K [2] & $\begin{array}{l}\text { two at 280m for flux and cross } \\
\text { sections, one identical at } 2 \mathrm{~km}\end{array}$ \\
NOvA [6] & $\begin{array}{l}1 \text { that is identical but moves } \\
\text { plus MINOS Near Detector } \\
\end{array}$ \\
& and MINERvA on axis \\
\hline \hline
\end{tabular}

\section{Muon Neutrino Disappearance}

Now that muon neutrino disappearance has been seen in an accelerator-based neutrino experiment at above three sigma [1], the next step is to measure the mass splitting that governs this disappearance precisely. This can only be done by seeing a variation of the disappearance probability as a function of neutrino energy. Different neutrino detectors measure neutrino energy differently, so uncertainties enter rather differently. We consider two kinds of energy measurements, and the ramifications for the measurement of oscillation parameters.

\subsection{Cerenkov Detectors}

Cerenkov detectors measure particles momenta and directions through The rings created by the cones of Cerenkov light emitted by particles above Cerenkov threshold. If an event shows the presence of only one outgoing muon, then the event can be classified as a muon neutrino quasi-elastic (QE) event, in which case the kinematics are completely specified (up to smearing due to Fermi motion in the nucleus). The incoming neutrino energy can be calculated by the simple formula

$E_{\nu}=\frac{m_{N} E_{\mu} m_{\mu}^{2} / 2}{m_{N} E_{\mu}+p_{\mu} \cos \theta_{\mu}}$

Where $E_{\mu}, p_{\mu}, \theta_{\mu}, m_{\mu}$ are the muons energy, momentum, direction and mass, respectively, and $m_{N}$ is the nucleon mass. 
However, the neutrino energy is misreconstructed in this technique when the event is in fact not a quasi-elastic event, but is a resonance or deep inelastic scattering event whose other final state particles are below Cerenkov threshold. The amount by which the energy is mis-reconstructed depends on the hadronic mass of the event, and therefore the overall neutrino energy scale uncertainty can be parameterized by uncertainty on the ratio of QE to non-QE events that are expected in the far detector event sample. Figure 1 shows the error on $\sin ^{2} 2 \theta_{2 \mathrm{sm}}$ and the mass splitting as a function of the mass squared splitting for both the statistical error after a five year run at $\mathrm{T} 2 \mathrm{~K}$ and the systematic shift induced by assuming a 10 or 20 per cent shift on the QE to non- $\mathrm{QE}$ ratio [11].
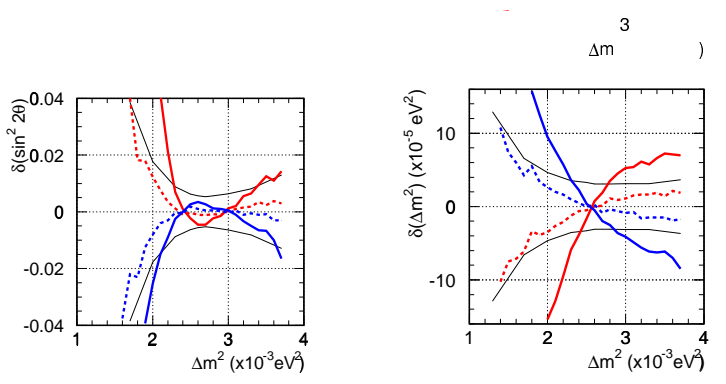

Figure 1. Statistical errors (thin black lines) and systematic shifts (thick colored lines) on the atmospheric mass splitting (left) and mixing angle (right) assuming a 10 and 20 per cent shift in the $\mathrm{QE}$ to non-QE ratio, for the $\mathrm{T} 2 \mathrm{~K}$ experiment after 5 years of running.

\subsection{Calorimetric Detectors}

For a calorimetric detector such as MINOS or $\mathrm{NOvA}$, the minimum threshold for pion and proton detection is much lower, and so much more of the final state energy may be visible in the detector-that is, once those particles escape from the nucleus. As the multiplicity of the final state particles increases (or as the energy of those particles decreases) then more and more energy is lost in the nucleus through pion rescattering and absorption. Figure 2 shows the fractional difference in reconstructed to true energies for QE, resonance, and deep inelastic scattering (DIS) events for MINOS for $3 \mathrm{GeV}$ neutrino events. Note that as the hadronic system mass of the event increases, the mean of the distribution decreases, and the RMS increases.
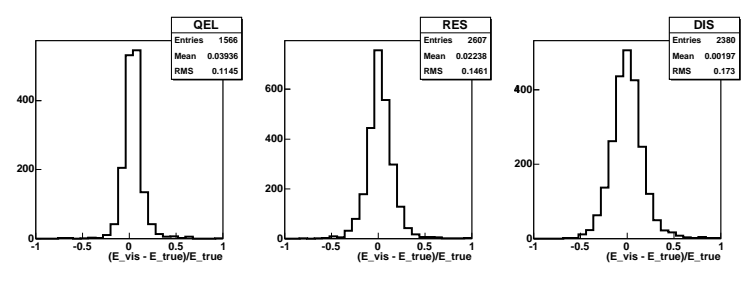

Figure 2. Fractional difference between true and visible energy for $3 \mathrm{GeV}$ neutrinos in the MINOS detector, for QE (left), Resonance (middle), and DIS (right) events.

So although these effects are much smaller than the loss of energy between quasi-elastic and nonquasi-elastic events, the processes that give rise to this extra energy loss in the nucleus are not wellknown, and therefore can result in uncertainties in the overall energy response of the detector. By comparing neutrino scattering off different nuclei one can constrain models of these nuclear effects. This is one of the goals of the upcoming MINERvA experiment [12].

\section{3. $\nu_{e}$ Appearance}

Measurements of the atmosperic neutrino oscillations and solar neutrino oscillations have shown that at least two of the mixing angles in the matrix that translates between mass and flavor eigenstates are large. If one assumes that there are only three generations that oscillate, then there is only one remaining mixing angle and a $\mathrm{CP}$-violating phase that remain to be measured to completely define this matrix. In three generations, the way to determine if this last mixing angle is non-zero, and to see if there is CP violation in the lepton sector, is to search for $\nu_{\mu} \leftrightarrow \nu_{e}$ 
Table 2

Survey of the different signal and background levels expected at for $\nu_{\mu} \rightarrow \nu_{e}$ searches, scaled to $\sin ^{2} 2 \theta_{13}=0.1 . \Delta m_{23}^{2}=2.5 \times 10^{-3} \mathrm{eV}^{2}$ for all predictions except for the $\mathrm{K} 2 \mathrm{~K}$ analysis which was for $\Delta m_{23}^{2}=2.8 \times 10^{-3} \mathrm{eV}^{2}$.

\begin{tabular}{lccccc}
\hline \hline & $\nu_{\mu}$ & $\nu_{e}$ & $\nu$ & $\nu_{\tau}$ & Osc. \\
Experiment & $\mathrm{CC}$ & Beam & $\mathrm{NC}$ & $\mathrm{CC}$ & $\nu_{e}$ \\
\hline K2K [13] & .25 & .4 & 1.7 & - & 0.8 \\
MINOS [14] & 4.7 & 4.7 & 20 & 1.7 & 15.5 \\
OPERA [15] & 1.0 & 18 & 5.2 & 4.5 & 9.3 \\
ICARUS [15] & - & 50 & - & 24 & 27 \\
T2K [11] & 0.7 & 13 & 9 & - & 103 \\
NOvA [6] & 0.5 & 12 & 7 & - & 142 \\
\hline
\end{tabular}

transitions at or near the atmospheric neutrino mass splitting frequency.

\subsection{Challenges}

The current and next generation long baseline experiments will have to face backgrounds from the intrinsic electron neutrino content of the beam as well as neutral (or even $\nu_{\mu}$ charged) current decays which get mis-reconstructed as electron neutrino charged current interactions. The extent to which the latter two occur is strongly dependent on discrimination between electrons and neutral pions, while the former is defined by beamline geometry and somewhat by detector energy resolution, and is in the next generation of experiments roughly a few tenths of a per cent. Finally, for the experiments which are operating above the $\nu_{\tau}$ charged current cross section threshold, the decay $\tau \rightarrow e$ can also provide a background to $\nu_{\mu} \rightarrow \nu_{e}$ appearance.

Clearly the smaller the oscillation probability is, the more precisely experiments need to determine these background predictions. However, the larger the oscillation probability is, the more important knowing the signal cross sections will be.

Table 2 gives a summary of the different backgrounds and possible signals for $\sin ^{2} 2 \theta_{13}=0.1$ for several different $\nu_{\mu} \rightarrow \nu_{e}$ searches. From this table one can see two different categories of experiments, depending on whether or not the back- grounds are dominated by the intrinsic $\nu_{e}$ content of the beam. K2K and MINOS are dominated by the neutral current (NC) backgrounds, and given the statistics that MINOS expects with $9 \times 10^{20}$ protons on target, an uncertainty of better than $20 \%$ would be required to have the statistics and systematics be equal. The second category of experiments are dominated by the intrinsic $\nu_{e}$ in the beam. In this case the background predictions can again be important if the signal seen is small, but for NOvA and $\mathrm{T} 2 \mathrm{~K}$ it is clear that if a big signal is seen, then to extract an oscillation probability the signal cross sections themselves will have to be precisely known.

As an example of how cross sections do not cancel completely between the near and far detectors, consider a study done on an earlier NOvA detector design (but one whose backgrounds were still dominated by intrinsic $\nu_{e}$ 's in the beam: if one assumes a near detector that has the same efficiency and background rejection as a far detector (which would already be hard to achieve given the different fiducial masses), one can vary the cross sections by their uncertainties and see how the ratio of far over near events changes as a function of the size of the $\nu_{\mu} \rightarrow \nu_{e}$ transition. Figure 3 gives the systematic and systematic uncertainties on the measured mixing angle (assuming no $\mathrm{CP}$ violation or matter effects, for simplicity) as a function of mixing angles for two different scenarios: the first is with current cross section uncertainty assumptions, the second is for assumed cross section uncertainties after the MINERvA experiment runs at Fermilab [16]. Although the detector designs have evolved since this early study, the differences between near and far detector event samples will still be significant and be an important motivation for precise cross section measurements.

\section{Information from Elsewhere}

It is clear from the above discussion that near detectors cannot measure all of the things that are needed to make precision oscillation probability measurements. Much work has gone into designing beam-lines that can accept large numbers of protons on the targets, but those designs must be 
6

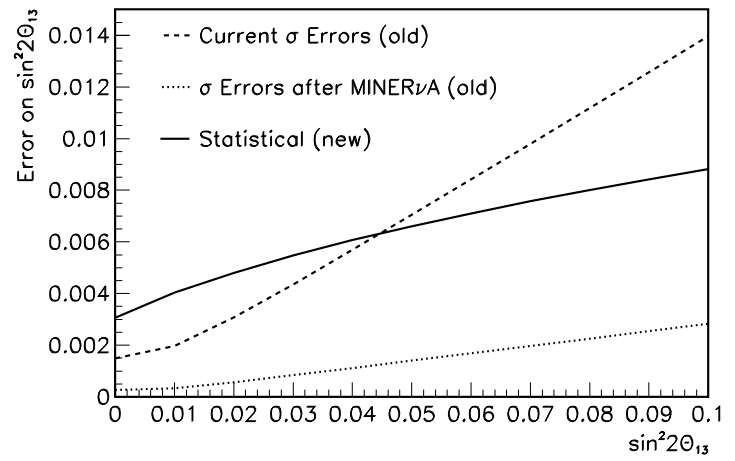

Figure 3. Uncertainty on the mixing angle $\sin ^{2} 2 \theta_{13}$ as a function of the angle itself for two different sets of assumptions on cross section uncertainties.

supported with precision measurements of hadron production and neutrino interactions (for example, by the MIPP [17] and MINERvA [12] experiments at Fermilab) in order to be able to take advantage of the increased statistics these protons could bring.

\section{Acknowlegements}

This article and the associated talk represent the work of many different people across several different experiments-Tsuyoshi Nakaya, David Petyt, Peter Litchfield, Kevin McFarland, Patricia Vahle and Tingjung Wang were all very helpful in pointing me towards their own or their collaborators' work. I am grateful for their help and continued communications.

\section{REFERENCES}

1. $\mathrm{K} 2 \mathrm{~K}$ Collaboration (E. Aliu et $a l$ ), Phys.Rev.Lett. 94 081802,2005

2. T2K Collaboration T. Nakadaira et al, Nucl.Phys.Proc.Suppl. 149303-305,2005

3. NuMI Technical Design Handbook, http://www-numi.fnal.gov/numwork/tdh/ tdh_index.html

4. CNGS web page, http:// proj-cngs.web.cern.ch/proj-cngs/

5. MINOS Technical Design Report, http : //www-numi . fnal.gov: 8875/Minos/ info/minos_tdr.html
6. NOvA Collaboration D.S.Ayres et al, hep-ex/0503053

7. Super-Kamiokande Collaboration M. Shiozawa et al, Nucl.Instrum.Meth.A433 240246,1999

8. OPERA Collaboration (G. Rosa et al.) Nucl.Phys.Proc.Suppl.145 98-101,2005

9. ICARUS Collaboration (S. Amerio et al.), Nucl.Instrum.Meth.A527 329-410,2004

10. K2K Collaboration, R. Gran et al, Nucl.Phys.Proc.Suppl.139, 54-58,2005

11. K. Hiraide and T. Nakaya, private communication

12. MINERvA proposal, D. Drakoulakos et al, hep-ex/0405002, Feb. 2004.

13. K2K Collaboration M.Ahn et al, Phys.Rev.Lett.93 051801,2004

14. For $9 \times 10^{20}$ protons on target, T. Yang, private communication and MINOS document 1143

15. M. Komatsu, P. Migliozzi, F. Terranova, J.Phys.G29 443,2003

16. MINERvA collaboration (D.A. Harris et $a l$ ), hep-ex/0410005, Oct. 2004.

17. H. Meyer for the MIPP collaboration, Nucl.Phys.Proc.Suppl.142 453-458,2005 